\title{
BUSINESS PERFORMANCE DECISIONS BY SOCIAL BUSINESS INTELLIGENCE
}

\author{
Doina FOTACHE \\ Alexandru Ioan Cuza University of Iasi \\ doina.fotache@feaa.uaic.ro \\ Ionuţ Daniel ANASTASIEI \\ Alexandru Ioan Cuza University of Iasi \\ ionut_daniel_anastasiei@yahoo.com \\ Vasile-Daniel PĂVĂLOAIA \\ Alexandru Ioan Cuza University of Iasi \\ danpav@uaic.ro
}

\begin{abstract}
The difficulty in interpreting social data through the usual software used by analysts has generated a new segment in the Business Intelligence (BI) market, namely Social Business Intelligence (SBI). The paper investigates the support of SBI in identifying the behavior of customers. Thus, this paper proposes an analysis of the SBI support that facilitates behavioral analysis, and as inputs it were used clicks, link accessing, or participation in online campaigns. The BI Domain supports Social Media (SM) analyzes and uses Extract Transform Load (ETL) technology to process and transform social data into data that can become the basis for business decisions. In this respect, two campaigns, namely conducted by e-mail and Facebook, were analyzed on the model of an insurance company. The aim was to identify which one, from an audience access point of view, is more efficient. The T test was used as a statistical tool, and this research was carried out based on the Facebook Business Manager package. The objective of this paper is to compare the indicators of the two modes of interaction (e-mail vs. Facebook) and to highlight the advantages of using SBI in companies.
\end{abstract}

Keywords: social business intelligence, social network analysis, ETL technologies JEL classification: M15, M31, M39

DOI: $10.12948 / \mathrm{ie} 2019.04 .12$

\section{Introduction}

Social networks directly influence consumers' behavior, generating new generations of consumers, with increasingly different behaviors and thus more difficult to anticipate due to the dynamics of the now-a-days times. Continuous customer monitoring provides a true picture of behavioral changes. In addition to structured data from business platforms, SM contributes with completely unstructured data, delivered in particular as comments. In this context, the most important issue is the analysis of users' comments and interactions. At this level, algorithm modeling is very difficult because comments can be difficult or even impossible to interpret, computer algorithms being "tricked" by certain words or groups of words. From the semantic point of view, the problems that appear are difficult because the social data are not perfect for different reasons: they are limited (the user sets the publication and its duration), incomplete (not always the location, gender or other relevant personal details) and many accounts are false (content invalid). 
www.conferenceie.ase.ro

\section{Literature review}

With the success of social platforms such as Twitter, Facebook, or Wikipedia, the "social" attribute quickly became a trend and was abusively used as a key word in many cases [1]. In order to overcome this situation, it can be found many efforts in the literature that aim at establishing a common scheme for defining and classifying social environments to allow judgments on what this concept implies [1], [2], [3], [4], [5]. However, the term social media is not fully clarified, and the definitions overlap social software with Web 2.0 [5], [2]. According to Global Web Index [6] over 37\% of Internet users follow favorite brands on SM channels, while another $30 \%$ use the SM to get information on certain products. All of these social data are unstructured. BI requires analysts to consider both existing data types, whether structured or unstructured [7]. It should be noted that data obtained from SM (social data) can be considered as semi-structured data simply because it can be categorized and can have parentchild relationships between values. At the same time, at the beginning of the 2000s it was considered that $60 \%$ of CIOs or CTOs agreed that unstructured data are critical to improving business processes and creating new opportunities. In anticipation of this review of literature, we could not find a unanimously accepted definition of SBI in scientific literature. One of the reasons could be the continued use of various terms, such as SM analytics, SM intelligence, social intelligence, and BI 2.0. We follow Zeng's understanding [8] that distinguishes between SM analytics and SM intelligence and defines the latter as: "SM intelligence aims to obtain actionable information from SM in the context of rich applications context, to develop decision-making frameworks or appropriate decision-making support, and to provide architectural projects and solutions frameworks for existing and new applications". The first steps in the development of Social BI were introduced on September 13, 2011, when Dachis Group created a social business index [9] which was intended to improve the vision of Big Data's analysis platforms. Demand for social features in BI solutions already exists in $59 \%$ of companies in 2014, given the "ability of users to share and collaborate" as one of the most important features when acquiring a BI solution, according to a report of the Information Week [10]. The merge between SM-BI [11] has occurred with the establishment of the target market where companies want to sell their products and services. Identifying this target market was an essential step for marketing plans [12], because it can segment the market by demographic factors, purchasing power and psychological factors [13], [14]. All of this has been gathered in the form of social data that is represented by the information that an individual creates and delivers voluntarily and deliberately to personal content in social media. Specialized techniques and tools have emerged for these data [15] including BI Models to Analyze SM data [16].

\section{Research methodology}

The analysis underlying the research methodology is approached from the Business Analyst perspective that uses statistical and BI tools in analyzing social data for marketing campaigns on SM. Figure 1 reflects the framing of the research methodology into the Data Science model. Once all the reference channels have been established, and at the same time the metrics that will provide a pertinent idea of the success or failure of the campaign, it was accessed the easiest way to obtain the data sources for analysis in Alteryx. Alteryx is the leader in selfservice data analytics and provides analysts with the unique ability to easily prep, blend and analyze all of their data using a repeatable workflow, then deploy and share analytics at scale for deeper insights in hours, not weeks. 


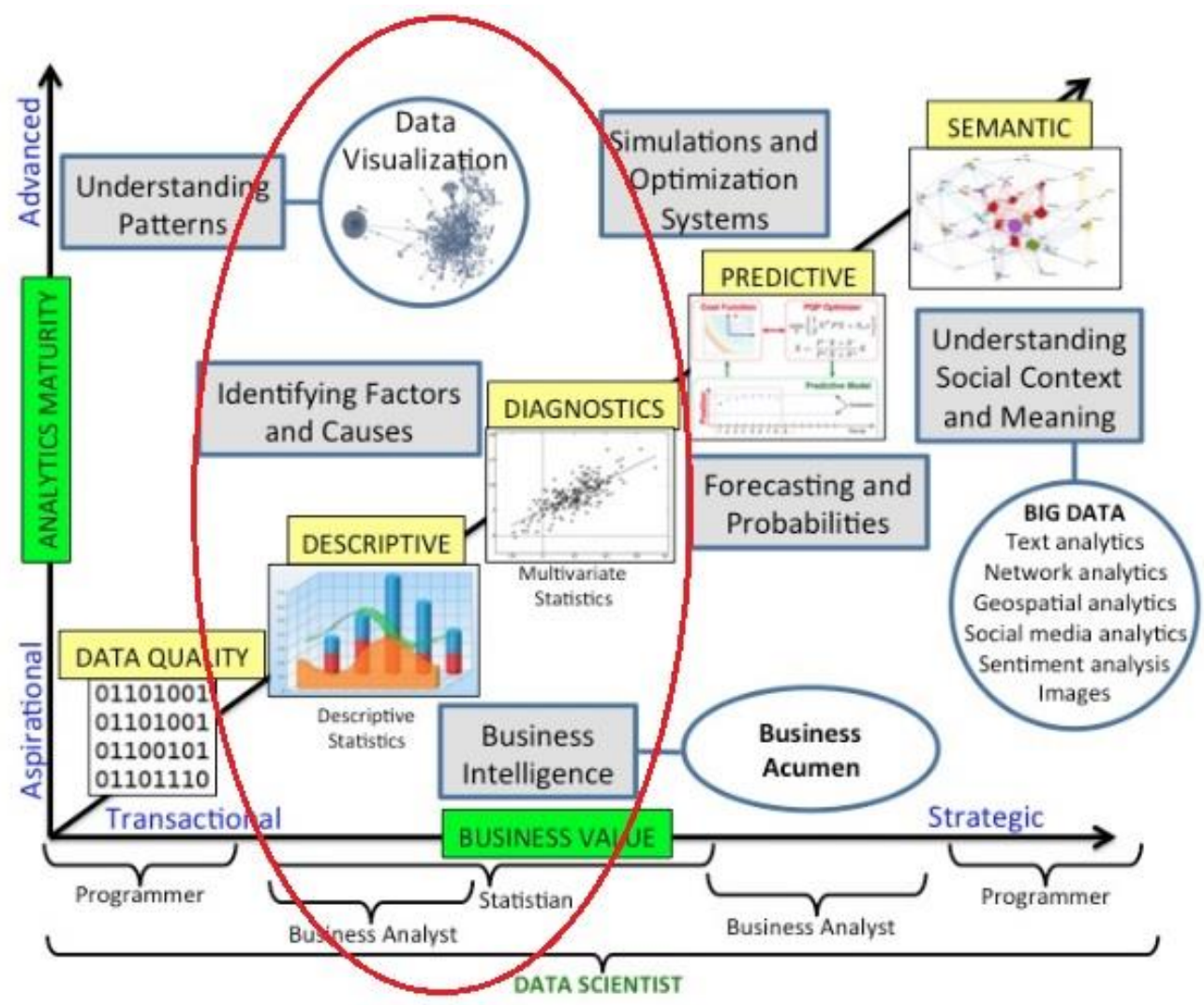

Figure 1. Framing the research methodology into the Data Science model Source: Granville, V., ,Data Science Summarized in One Picture”,

https://www.datasciencecentral.com/profiles/blogs/data-science-summarized-in-one-picture

After running the ETL process from Alteryx, the T test is applied on the data that refers to the effectiveness of an online campaign. Therefore, the statistical significance was analyzed using $\mathrm{T}$ test which is generally applied to normal distribution data which has a small set of values. As such, the data was first tested for normal distribution and as the result was positive, the $\mathrm{T}$ test was applied on the set of data in order to test the Hypotheses and validate the results.

The main hypothesis of the study is Social Networks have a considerable impact on promoting marketing companies, significantly more important than e-mail campaigns. Furthermore, the hypothesis has been broken down into six secondary hypotheses, namely:

H1: There are significant differences in the impact of the campaign on Facebook and email campaign

$\mathrm{H} 2$ : There are significant differences in the impact of the campaign on Facebook and email campaign by top five states in the US

H3: There are significant differences in the impact of the campaign on Facebook and on mail campaign based on the number of employees

H4: There are significant differences in the impact of the campaign on Facebook and on mail campaign based on years of service

H5: There are significant differences in the impact of the campaign on Facebook and on mail campaign based on turnover

H6: There are significant differences in the impact of the campaign on Facebook and email campaign by the industry

To demonstrate the main hypothesis, statistical t-test was used to fundament the relevance of information sampling and contact segmentation by results. The tests results are presented in 
www.conferenceie.ase.ro

two states, accepted and rejected, and have as an index of acceptance the index and the result of the statistical significance.

\subsection{Data collection}

The company for which this case study was conducted activates in the industry of insurance policies sale for US legal entities. In the problem discovery process, an information campaign was first launched on the Facebook Social Network where a US target market was considered. The market was derived from the company's existing customer portfolio. The selection of Facebook platform to conducting this research was based on the Facebook Business Manager package that allows the company to retrieve contacts (which is not possible for other SM platforms). Although the customer portfolio was initially 4000 contacts, due to Facebook Business Manager, the customer portfolio can be increased to almost 2.6 million contacts based on a $99 \%$ similarity rate.

\subsection{Instrument design}

In order for a company to understand the behavior of its clients on the social networking channels, it's necessary to analyze the behavior of the clients in two major manners: information campaign on Facebook and information campaign by e-mail.

The objective of this paper is to analyze comparatively the indicators of the two ways of interaction and highlight the advantages of using Social BI in companies.

The most difficult and complex component of the ETL process involved in the processing of undisclosed information was the preparation of statistical data.

After running the ETL process in the Alteryx program, the t test is applied to analyze how effective the campaign was in the online environment. The confidence levels presented in Table 1 represent the basis on which the statistical result is accepted or not.

Table 1. Confidence level in the result of statistical significance ( $t$-Test)

\begin{tabular}{|l|l|}
\hline Confidence level & Percent \\
\hline Low & $<80 \%$ \\
\hline Low-Medium & $>=80 \%$ \\
\hline Medium & $>=90 \%$ \\
\hline Medium-High & $>=95 \%$ \\
\hline High & $>=99 \%$ \\
\hline
\end{tabular}

The information in Table 1 and Figure 2 is closely related because, through the rules, statistical significance is relevant to the results obtained.

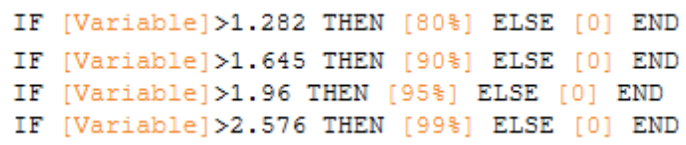

Figure 2. IF clause (represented by the Tableau program)

If the [Variable], which is the result of the $t$ test, is framed in the four parameters, it can be assigned to one of the confidence levels given to the results and can be statistically accepted only if the index is greater than or equal to 100 .

Figure 3 displays the model, developed in Excel, and used to automate reporting. This automation occurred with the difficult process of calculating each segment of analysis. At the 
same time, we can view the number of interactions that the Facebook campaign has had, depending on the industry in which contacts activate.

In Tableau, translating the fields used in Excel makes the formula easier to understand. Specifically, in Figure 3 we replaced the key fields and belonging to Figure 2, and thus the formula for calculating the $t$ test was applied to the data set.
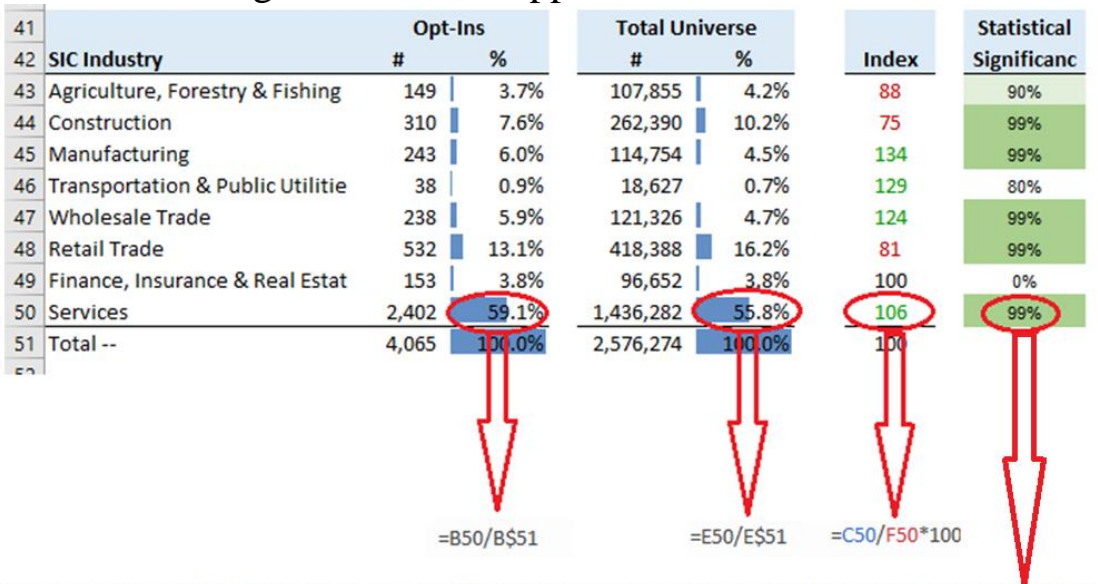

\author{
$\%$ confidence level \\ $95 \%$ confidence level \\ $90 \%$ confidence level \\ $<90 \%$ confidence level
}

$\operatorname{MAX}\left(I F(A B S)((B 50 / B \$ 51)-(E 50 / E \$ 51)) / S Q R T\left(\left(\left(B \$ 51^{*}(B 50 / B \$ 51)+E \$ 51^{*}(E 50 / E \$ 51)\right) /(B \$ 51+E \$ 51)\right)^{*}\left(1-\left(B \$ 51^{*}(B 50 / B \$ 51)+E \$ 51^{*}(E 50 /\right.\right.\right.\right.$ $\left.\left.\left.\$ 51)) /(B \$ 51+E \$ 51) * *(B \$ 51+E \$ 51)) /\left(B \$ 51^{*} E \$ 51\right)\right)\right)>=1.282,80 \%, 0 \%\right), I F\left(A B S\left(((B 50 / B \$ 51)-(E 50 / E \$ 51)) / S Q R T\left(\left(\left(B S 51^{*}(B 50 / B \$ 51)+E \$ 51^{*}\right.\right.\right.\right.\right.$ $\left.\left.\left.\left.50 / E \$ 51)) /(B \$ 51+E \$ 51))^{*}\left(1-\left(B \$ 51^{*}(B 50 / B \$ 51)+E \$ 51^{*}(E 50 / E \$ 51)\right) /(B \$ 51+E \$ 51)\right)^{*}(B \$ 51+E \$ 51)\right) /\left(B \$ 51^{*} E \$ 51\right)\right)\right)>=1.645,90 \%, 0 \%\right), I F(A B S((1$ i50/B\$51)-(E50/E\$51))/SQRT((((B\$51*(B50/B\$51)+E\$51* $(E 50 / E \$ 51)) /(B \$ 51+E \$ 51))^{*}\left(1-\left(B \$ 51^{*}(B 50 / B \$ 51)+E \$ 51^{*}(E 50 / E \$ 51)\right) /(B \$ 51+\right.$ $\left.\left.\left.\left.\$ 51))^{*}(B \$ 51+E \$ 11)\right) /\left(B \$ 51^{*} E \$ 51\right)\right)\right)>=1.96,95 \%, 0 \%\right), I F\left(A B S\left(((B 50 / B \$ 51)-(E 50 / E \$ 51)) / S Q R T\left(\left(\left(B \$ 51^{*}(B 50 / B \$ 51)+E \$ 51^{*}(E 50 / E \$ 51)\right) /(\right.\right.\right.\right.$ $\left.\left.\left.\left.\left.\$ 51+E \$ 51))^{*}\left(1-\left(B \$ 51^{*}(B 50 / B \$ 51)+E \$ 51^{*}(E 50 / E \$ 51)\right) /(B \$ 51+E \$ 51)\right)^{*}(B \$ 51+E \$ 51)\right) /\left(B \$ 51^{*} E \$ 51\right)\right)\right)>=2.576,99 \%, 0 \%\right)\right\}$

Figure 3. Graphical representation of index formulas / statistical significance in Excel

\title{
3. Results and discussions
}

\subsection{Metrics analysis for Facebook campaign}

The following are the results of the statistical significance test and also the acceptance / rejection of the results according to the index. We mention that a test should be considered only if the index is greater than or equal to 100 and if the statistical result is at least $90 \%$.
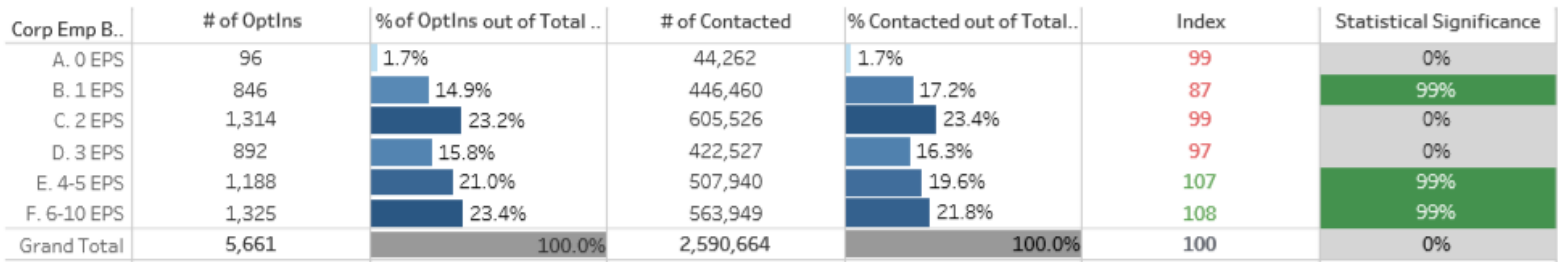

Figure 4. Statistical significance by number of employees

\begin{tabular}{|c|c|c|c|c|c|c|}
\hline Total Sales Bucket & \# of OptIns & \%of Optins out of Total ... & $\#$ of Contacted & $\%$ Contacted out of Tota.. & Index & Statistical Significance \\
\hline c. $\$ 5 \mathrm{~K}-\$ 50 \mathrm{~K}$ & 428 & $7.6 \%$ & 224,940 & $8.7 \%$ & 87 & $99 \%$ \\
\hline E. $\$ 100 K-\$ 250 K$ & 1,667 & $29.4 \%$ & 770,469 & $29.7 \%$ & 99 & $0 \%$ \\
\hline F. $\$ 250 K-\$ 500 K$ & 1,159 & $20.5 \%$ & 491,872 & $19.0 \%$ & 108 & $99 \%$ \\
\hline G. $\$ 500 K-\$ 1 M M$ & 821 & $14.5 \%$ & 358,392 & $13.8 \%$ & 105 & $80 \%$ \\
\hline
\end{tabular}

Figure 5. Statistical significance by turnover 
www.conferenceie.ase.ro
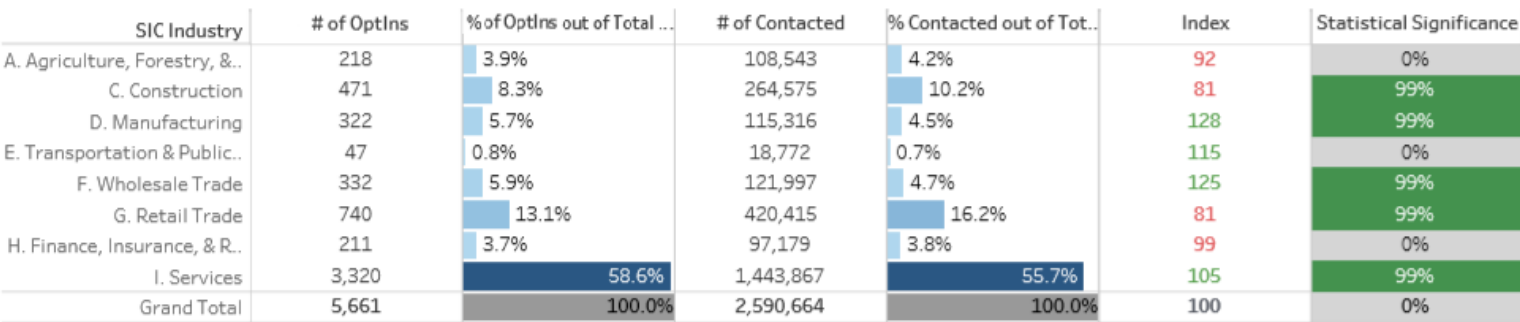

Figure 6. Statistical significance by field of activity

Table 2. Testing social campaign results

\begin{tabular}{|c|c|c|c|c|}
\hline Sector analyzed & Analyzed metric & Index (i) & Statistical significance (S) & Result \\
\hline \multirow{3}{*}{ Employee number } & 1 employee & 87 & $99 \%$ & Reject \\
\hline & 4-5 employees & 107 & $99 \%$ & Accept \\
\hline & 6-10 employees & 108 & $99 \%$ & Accept \\
\hline \multirow{3}{*}{ Turnover } & $\$ 5 \mathrm{k}-\$ 50 \mathrm{k}$ & 87 & $99 \%$ & Reject \\
\hline & $\$ 50 \mathrm{k}-100 \mathrm{k}$ & 93 & $95 \%$ & Reject \\
\hline & $\$ 250 \mathrm{k}-\$ 500 \mathrm{k}$ & 108 & $99 \%$ & Accept \\
\hline \multirow{5}{*}{ Industry } & Constructions & 81 & $99 \%$ & Reject \\
\hline & Services & 105 & $99 \%$ & Accept \\
\hline & Transport & 115 & $99 \%$ & Accept \\
\hline & Wholesale sales & 125 & $99 \%$ & Accept \\
\hline & Manufacturing & 128 & $99 \%$ & Accept \\
\hline
\end{tabular}

\subsection{Metrics analysis for the e-mailing campaign}

In the e-mailing campaigns, as well as in Facebook's, companies with employees number between 6-10 had a fairly high e-mail response rate.
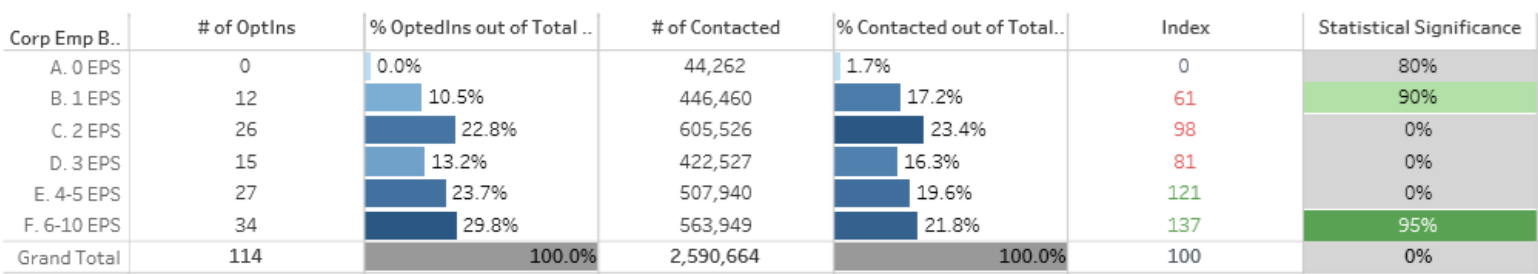

Figure 7. Statistical significance of marketing campaigns by number of employees

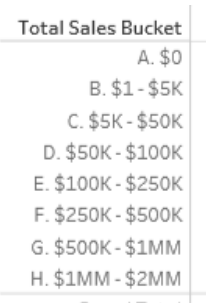

\# of Optins
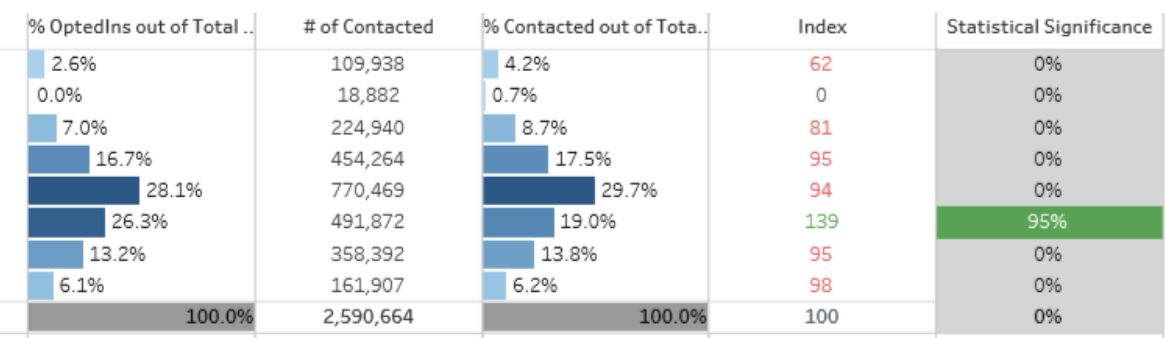

Figure 8. Statistical significance of marketing campaigns based on turnover 
www.conferenceie.ase.ro
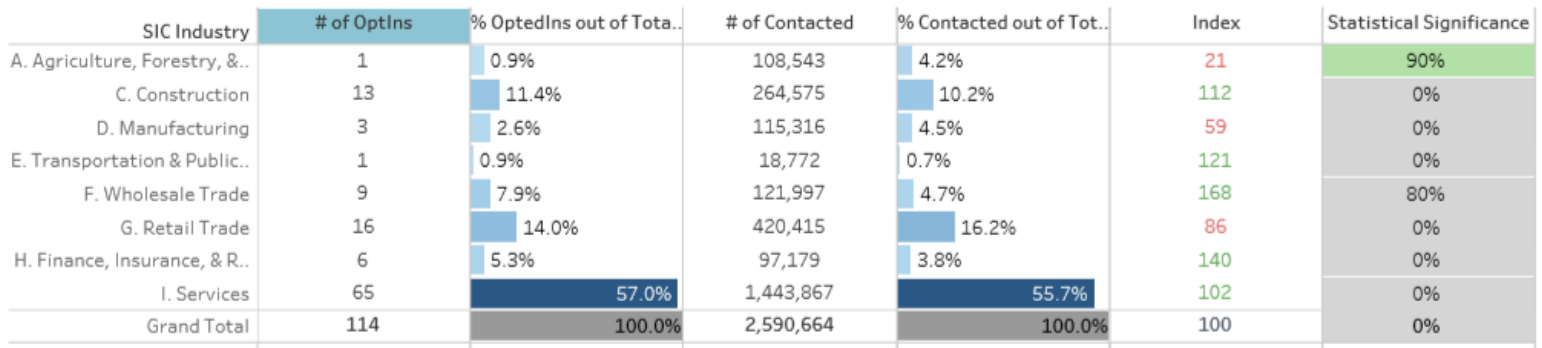

Figure 9. Statistical significance by industry in marketing automation campaigns

Analyzing the results presented in Tables 2 and 3 and Figures 4-9 it can be seen that the results of the Facebook campaign were clearly superior to those of the e-mail. This does not just refer to the number of companies that visited the campaign site, but also to the number of accepted test cases, namely seven against two, in favor of Facebook social networking.

Table 3. Testing Results for e-mailing campaign

\begin{tabular}{|l|l|l|l|l|}
\hline Sector analyzed & Analyzed metric & Index (i) & Statistical significance (S) & Result \\
\hline Employee number & 1 employee & 61 & $90 \%$ & Reject \\
\cline { 2 - 6 } & $6-10$ employees & 137 & $95 \%$ & Accept \\
\hline Turnover & \$250k-\$500k & 139 & $95 \%$ & Accept \\
\hline Industry & Agriculture & 21 & $95 \%$ & Reject \\
\hline
\end{tabular}

BI tools along with performance metrics and SM contacts should be used to meet companies' business goals. Metrics such as the number of clicks, the number of visits to the campaign, or the number of views are not enough to invalidate or confirm the success of a campaign. In this situation, the statistical significance comes in, contributes toward distinguishing between the company's truly important data and the data that could be directed to be used previously.

The two campaigns, conducted in Facebook and e-mailing, have different approaches to prospecting the market and capturing new customers. Facebook uses an indirect advertising approach, while the mailing campaign is based on a direct approach through the Inbox.

In this research, we started from building the company database for the two campaigns, as well as collecting the market segment results in order to be able to track the activity of the contacts on the campaign page, the results obtained are summarized below, where $\mathrm{Rx}$ - results, $\mathrm{Hx}$ secondary hypothesis, Dx - decision:

- R1: According to the analysis, 5,661 contacts responded to the Facebook campaign, while only 114 interacted with our mailing page.

- H1: The Facebook campaign had a considerably larger impact than the mail, with a difference that is being considerable.

- D1: The decision in this case can only consider the Facebook campaign in the case the marketing department intends to promote the company.

- R2: In terms of employee-related contacts, three sizes of companies were tested within the campaigns. Of them, two are accepted: companies with 4-5 employees and companies with 6-10 employees. In the case of the mail campaign, two sizes of companies were used. From them, only one was accepted, namely the one with 6-10 employees.

- H2: Both campaigns have had statistically significant results, but trust goes further on the SM campaign, given that two tests have been accepted.

- D2: The business decision is that both campaigns had a good score in the 6-10 sector. As such the target group will be in the next campaign. 
www.conferenceie.ase.ro

- R3: The business-to-business contacts segment had three SM test cases, with companies with an income ranging between 250,000-500,000 US dollars. By mail, the statistical test had similar result.

- H3: Both campaigns have provided valuable results for the following campaigns, by achieving the same score. However, in the next campaigns, the two other statistical tests that had a weak index could be attempted.

- D3: In the process of shaping the target group it shall be considered companies with turnover between 250,000 and 500,000 USD.

- R4: For segmented contacts according to the domain of activity, there were five test cases in the Facebook campaign, of which three were accepted. In the mail campaign, a single test was performed but was not accepted.

- H4: The contacts for the sectors: production, wholesale trade and services are accepted as the results have proved to be statistical significant.

- D4: The ideal client can be found in one of the three sectors mentioned in H4 and will be targeted in the following campaigns.

Sharpening the target group according to the results obtained from the research, considers the following scenario: According to the $\mathrm{H} 2, \mathrm{H} 3, \mathrm{H} 4$ hypotheses, the ideal client profile operates in California, has 6 to 10 employees, works in the field of production, wholesale or service, and has an annual turnover of between 250,000 and 500,000 USD.

According to D1, D2, D3, D4, the next marketing campaign will take place on Facebook and will target a client with the above characteristics.

The final conclusion of this paper is that BI platforms can collect and interpret SM data and provide decision support that will generate multiple opportunities for a company. Practice can be used by any company regardless of the industry. In this regard, the work reveals that the support provided by BI technologies for the purpose of collecting and interpreting the results is essential.

\section{Future paths}

In a wider research, contact's reactions to a campaign can also be measured by an extensive analysis across other social networks, not just Facebook. In the current research only the initial campaign phase and target group setting were considered. Also, it was determined the campaign's feasibility score. However, at a later stage, a new campaign can be performed, applied only on contacts that have managed to get a better score according with the statistical significance.

Another perspective could be that of gathering information about market segments whose results were good, but the index and the result of statistical significance were rejected. It is a contradiction between the two, so one can thoroughly investigate what was wrong in order to attract other potential customers.

At the same time, in the future, it shall be included the users' responses from all social networks, as well as the number of likes, the number of comments or distributions to the campaign. Such action can provide the company with a broader view of the social environment.

\section{References}

[1] J. Kietzmann, K. Hermkens, I. McCarthy, B. Silvestre, "Social media? Get serious! Understanding the functional building blocks on social media", Business Horizons, vol. 54, no. 3, 2011, pp. 241-251.

[2] A. M. Kaplan, M. Haenlein, "Users of the world, unite! The challenges and opportunities of Social Media”, Business Horizons, vol. 53, no. 1, 2010, pp. 59-68. 
[3] D. M. Boyd, N. B. Ellison, "Social Network Sites: Definition, History and Scholarship", Journal of Computer-Mediated Communication, vol. 13, no. 1, pp. 210-230, 2007.

[4] M. Parameswaran, A. B. Whinston, "Research Issues in Social Computing", Journal of the Association for Information Systems, vol. 8, no. 6, pp. 336-350, 2007.

[5] T. O'Reilly, "What is Web 2.0: Design patterns and business models for the next generation of software", Communications \& strategies, vol. 17, no. 1, 2007.

[6] M. Jason, Trends 2016: Social Commerce, 2016, https://blog.globalwebindex.com/chartof-the-day/trends-2016-social-commerce [Accessed: Jan, 2019].

[7] K. Rudin, D. Cressy, "Will the Real Analytic Application Please Stand Up?" DM Review, vol. 13, no. 3, pp. 30-34, 2003.

[8] D. Zeng, H. Chen, R. Lusch, S. Li, "Social Media Analytics and Intelligence," IEEE

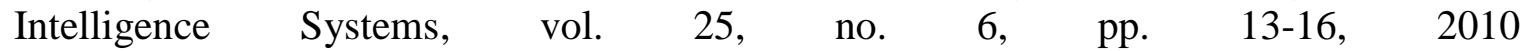
http://ieeexplore.ieee.org/lpdocs/epic03/wrapper.htm?arnumber=5678581 [Accessed: Jan, 2019].

[9] Social Business Index - Think Of It As Klout For Companies, 2011, https://techcrunch.com/2011/09/13/dachis-group-debuts-social-business-index-think-ofit-as-klout-for-companies/ [Jan, 2019].

[10] Social BI for Intelligent Enterprise $2.0 \quad 2014$, https://forteconsultancy.wordpress.com/2014/11/12/social-bi-for-intelligent-enterprise-20/ [Febr, 2019].

[11] Y. Lu, F. Wang, R. Maciejewski, "Business intelligence from social media: A study from the VAST box office challenge", IEEE Computer Graphics and Applications, vol. 34, no. 5, pp. 58-69, 2014.

[12] W. He, W. Harris, G. Yan, V. Akula, J. Shen, "A novel social media competitive analytics framework with sentiment benchmarks". Information \& Management, vol. 52, no. 1, 2015, pp. 801-812.

[13] S. Moro, P. Rita, B. Vala, "Predicting social media performance metrics and evaluation of the impact on brand building: A data mining approach", Journal of Business Research, vol. 69, no. 9, pp. 3341-3351, 2016.

[14] M. Harrysson, E. Metayer, H. Sarrazin, "How 'social intelligence' can guide decisions", McKinsey Quarterly, 2012.

[15] B. Batrinca, P. C. Treleaven, "Social media analytics: a survey of techniques, tools and platforms", Artificial Intelligence \& Society, vol. 30, no. 1, 2015, pp. 89-116.

[16] F. Parama, K. Suharjito, "Business Intelligence Model to Analyse Social Media Information," 2018, retrieved from Sciencedirect.com [Accessed: Jan, 2019]. 